\title{
Editorial
}

\section{Metal Oxide Heterostructures for Water Purification}

\author{
Xiang Wu, ${ }^{1}$ Xijin Xu, ${ }^{2}$ Chuanfei Guo, ${ }^{3}$ and Haibo Zeng ${ }^{4}$ \\ ${ }^{1}$ Key Laboratory for Photonic and Electronic Bandgap Materials, Ministry of Education and College of Chemistry \\ and Chemical Engineering, Harbin Normal University, Harbin 150025, China \\ ${ }^{2}$ School of Physics and Technology, University of Jinan, Jinan 250022, China \\ ${ }^{3}$ Department of Physics, University of Houston, Houston 77004, USA \\ ${ }^{4}$ School of Materials Science and Engineering, Nanjing University of Science and Technology, Nanjing 210094, China
}

Correspondence should be addressed to Xiang Wu; wuxiang05@gmail.com

Received 4 May 2014; Accepted 4 May 2014; Published 14 May 2014

Copyright ( $) 2014$ Xiang Wu et al. This is an open access article distributed under the Creative Commons Attribution License, which permits unrestricted use, distribution, and reproduction in any medium, provided the original work is properly cited.

In recent years, the toxic and colored wastewater containing dyes seriously affect quality of water, inhibit sunlight penetration, and reduce photosynthetic reaction. The previous techniques dealing with dye wastewater are usually inefficient and costly. At present, heterostructured semiconductor oxide photocatalysts have been regarded as very efficient and inexpensive catalysts to remove the dye pollutants from water due to their large specific surface areas and unique spatial architectures [1-4]. The hybrid semiconductor systems can promote the separation of electron-hole pairs and keep reduction and oxidation reactions at two different reaction sites, which improves tremendously photocatalytic efficiency of semiconductor nanostructures.

As guest editors, we successfully organized a special issue titled "Self-assembly of semiconductor metal oxide nanostructures" in Journal of Nanomaterials in the beginning of this year [5]. Now we are pleased to finish this special issue on metal oxide heterostructures for water purification. This special issue covers all topics related to metal oxide nanomaterials towards the treatment, purification, and remediation of surface water, groundwater, and industrial wastewater streams. This special issue consists of two review papers and eight research papers that are contributed from Chinese scientists.

The review article by L. Chen et al. summarized metal oxide heterostructures for arsenic removal from contaminated water to provide efficient, economic, and robust solutions for water purification. They gave a list of various metal oxides, including activated alumina, iron oxide, titanium oxide, zirconium oxide, manganese oxide, binary metal oxides, and metal oxide heterostructures and present a broad view of metal oxide heterostructures as the adsorbents that have been identified for use in arsenic removal from water systems. Q. Zhang et al. reviewed most recent activities in $\mathrm{TiO}_{2}$ nanostructures with an emphasis on the authors' own results especially on those synthesized using anodic oxidation method. The review begins with a survey of the effects of fabrication methods and the experiment conditions on obtained $\mathrm{TiO}_{2}$ nanostructures and then focuses on syntheses, characterizations, formation mechanisms, and photocatalytic properties of their 1D nanostructures. Finally, they conclude this review with the perspectives and outlooks on the future developments in this field.

The article by Y. Zhang et al. reported template-free synthesis of porous $\mathrm{Cu}_{2} \mathrm{O}$ nanospheres at room temperature and systematic investigation on their adsorption properties. Their experiment results showed that the as-synthesized porous $\mathrm{Cu}_{2} \mathrm{O}$ nanospheres were composed of small nanoparticles with average diameters of $15 \mathrm{~nm}$. The adsorption ability of the as-prepared products towards methyl orange as the pollutant was investigated. In addition, they also studied the reusability in degradation methyl orange of the as-prepared products. By using a natural tree pollen grain as the template and sol-gel technology, X. Yang et al. synthesized heterogeneous catalytic materials based on polyoxometalate compounds with core-shell structure. The results show that the catalysts have excellent catalytic activity in treatment of wastewater. The catalyst under different cycling runs shows good stability under such operating conditions and the leaching tests show negligible leaching effect owing to the lesser dissolution. B. Yin et al. successfully synthesized high-quality nanoscale ultrathin $\mathrm{ZnO}$ films at relatively low temperature using a 
facile and effective hydrothermal approach. The products demonstrated $95 \%$ photodegradation efficiency with Congo red after $40 \mathrm{~min}$ irradiation, revealing that the as-obtained $\mathrm{ZnO}$ films might be promising candidates as the excellent photocatalysts for elimination of wastewater. Z. Chen et al. reported fabrication of $\mathrm{TiO} / \mathrm{TiO}_{2}$ heterogeneous nanostructures by employing high-energy ball-milling method. Photocatalytic degradation experiments revealed that an appropriate $\mathrm{TiO}$ content can enhance the photocatalytic activity of pure anatase $\mathrm{TiO}_{2}$ to a level better than that of Au-deposited $\mathrm{TiO}_{2}$ by keeping high degradation efficiency of $93 \%$. They thought the internal electrical field produced by $\mathrm{TiO} / \mathrm{TiO}_{2}$ heterogeneous nanostructures was dominantly responsible for the enhanced photocatalytic activity.

B. Zhou et al. synthesized $\mathrm{Cu}_{2} \mathrm{O} / \mathrm{Cu}$ hollow spheres using one-pot template-free solvent-thermal route. And the content of $\mathrm{Cu}$ in the hollow spheres can be easily controlled by adjusting the solvent-thermal synthesis time. The prepared $\mathrm{Cu}_{2} \mathrm{O} / \mathrm{Cu}$ hollow sphere exhibited a higher photodegradation capability than the corresponding nanoparticles and solid spheres. X. Zhang et al. reported fabrication of perfect cuprous oxide $\left(\mathrm{Cu}_{2} \mathrm{O}\right)$ octahedra by a facile route without chemical additive. They studied the adsorption behavior of the as-synthesized $\mathrm{Cu}_{2} \mathrm{O}$ octahedral in detail. X. Li et al. synthesized graphene oxide thin film by the mixture GO spin coated on substrate of indium tin oxide. The experiment results showed that continuous-wave laser is modulated when graphene oxide saturable absorber is employed in the 1064 $\mathrm{nm}$ laser cavity with the shortest pulse width of $108 \mathrm{~ns}$. Their results demonstrated that graphene oxide is an available saturable absorber for $1064 \mathrm{~nm}$ passive Q-switching laser. At last, Wu's group prepared large scale $\mathrm{ZnO}$ microflowers assembled by numerous nanosheets through a facile and effective hydrothermal route. Their results demonstrated that eosin red aqueous solution can be degraded over 97\% after 110 min under UV light irradiation.

\section{Acknowledgment}

As guest editors, we would like to thank all of the above authors for their efforts to this special issue. We are also grateful to the selfless referees for their hard work in reviewing these articles. We hope that these excellent contributions can provide a valuable reference to the current development and future research of metal oxide heterostructures for water purification. We also hope that the collection of these articles will inspire researchers and stimulate new ideas in relative research field.

$$
\begin{array}{r}
\text { Xiang Wu } \\
\text { Xijin Xu } \\
\text { Chuanfei Guo } \\
\text { Haibo Zeng }
\end{array}
$$

Science of Advanced Materials, vol. 5, no. 10, pp. 1364-1371, 2013.

[2] W. N. Jia, B. X. Jia, F. Y. Qu, and X. Wu, "Towards a highly efficient simulated sunlight driven photocatalyst: a case of heterostructured $\mathrm{ZnO} / \mathrm{ZnS}$ hybrid structure," Dalton Transactions, vol. 42, pp. 14178-14187, 2013.

[3] J. Tian, Y. H. Sang, G. W. Yu, H. D. Jiang, X. N. Mu, and H. Liu, "A $\mathrm{Bi}_{2} \mathrm{WO}_{6}$-based hybrid photocatalyst with broad spectrum photocatalytic properties under UV, visible, and near-infrared irradiation," Advanced Materials, vol. 25, no. 36, pp. 5075-5080, 2013.

[4] J. Tian, Y. H. Sang, Z. H. Zhao et al., "Enhanced photocatalytic performances of $\mathrm{CeO}_{2} / \mathrm{TiO}_{2}$ nanobelt heterostructures," Small, vol. 9, no. 22, pp. 3864-3872, 2013.

[5] X. Wu, X. J. Xu, H. B. Zeng, and C. F. Guo, "Self-assembly of semiconductor metal oxide nanostructures," Journal of Nanomaterials, vol. 2013, Article ID 459516, 2 pages, 2013.

\section{References}

[1] J. Wang, F. Y. Qu, and X. Wu, "Photocatalytic degradation of organic dyes with hierarchical $\mathrm{Ag}_{2} \mathrm{O} / \mathrm{ZnO}$ heterostructures," 

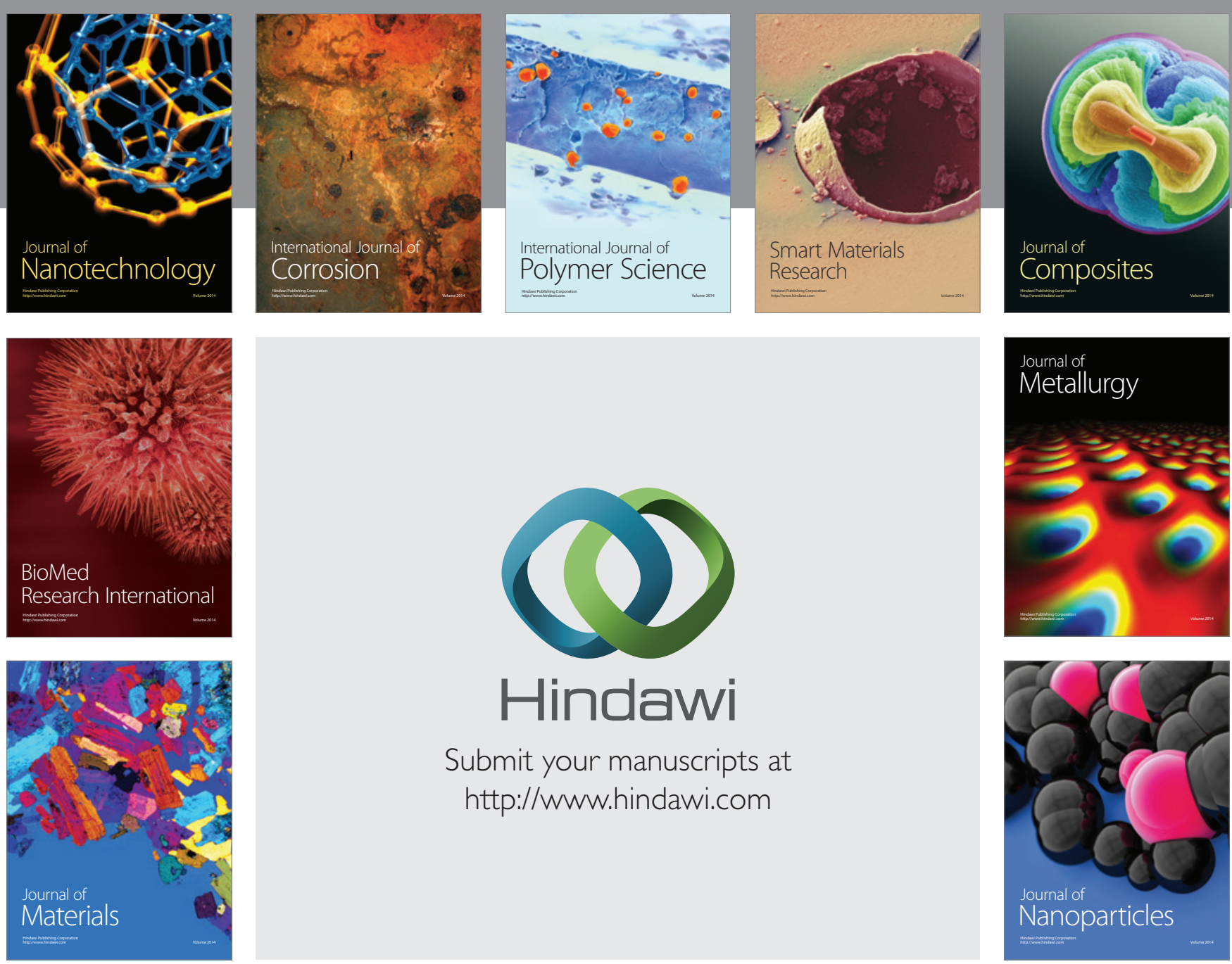

Submit your manuscripts at http://www.hindawi.com
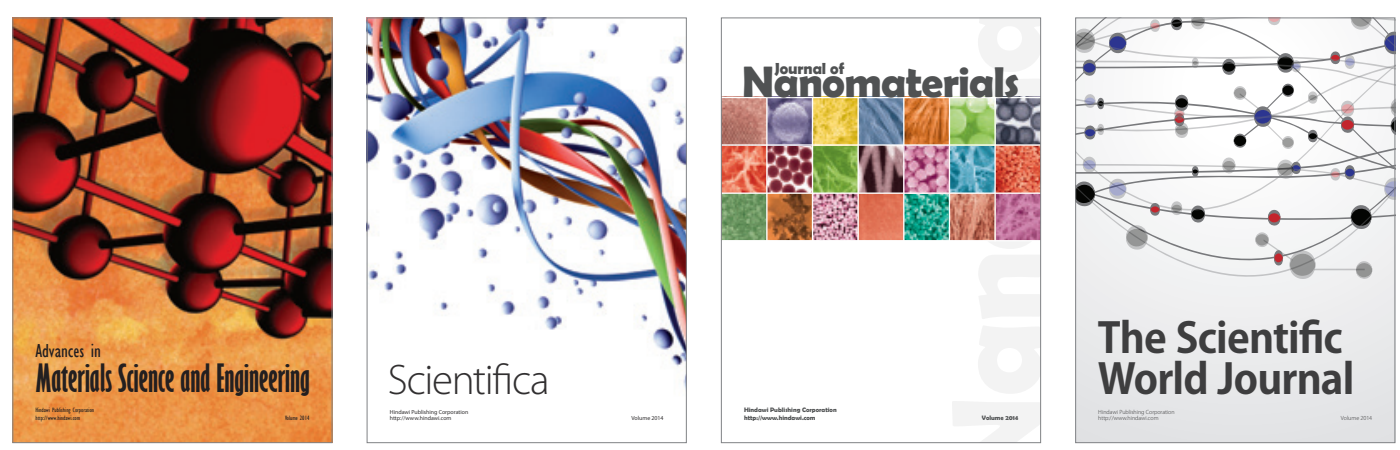

\section{The Scientific World Journal}
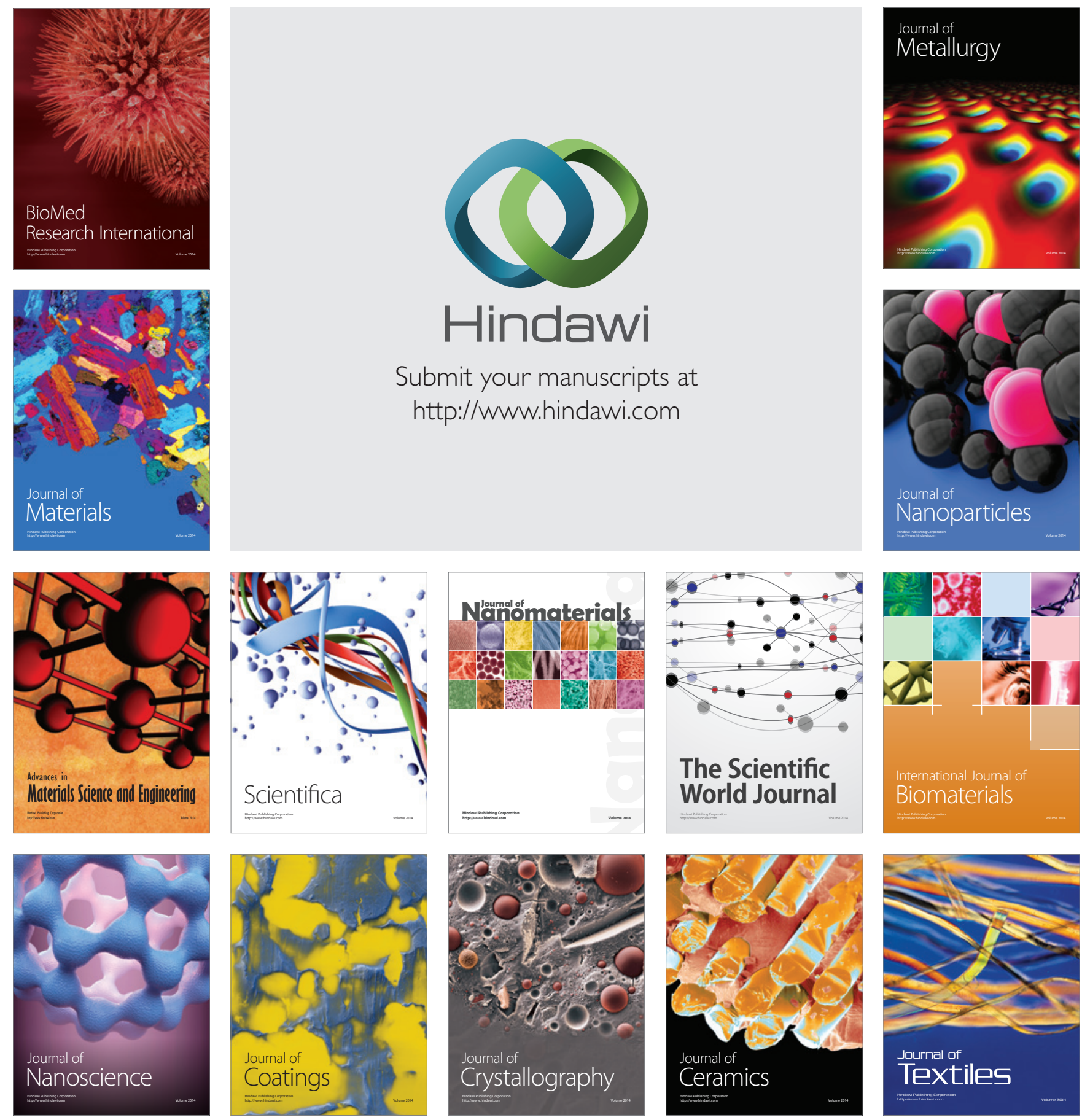